\title{
Surface-Focused Seismic Holography of Sunspots: II. Expectations from Numerical Simulations Using Sound-Speed Perturbations
}

\author{
A.C. Birch • D.C. Braun • S.M. Hanasoge $\cdot$ R. Cameron
}

Received: 5 October 2007 / Accepted: 21 October 2008 / Published online: 7 November 2008

(C) The Author(s) 2008. This article is published with open access at Springerlink.com

\begin{abstract}
Helioseismic observations of sunspots show that wave travel times, at fixed horizontal phase speed, depend on the temporal frequency of the waves employed in the data analysis. This frequency variation has been suggested to be consistent with near-surface (vertical length scales of order one Mm or smaller) changes in wave propagation properties relative to the quiet Sun. We investigate this suggestion by employing numerical simulations of acoustic-wave propagation through models with horizontally and vertically inhomogeneous structure. Standard methods of surface-focused helioseismic holography are applied to the resulting simulated wave fields. We find that the travel-time shifts measured using holography from the simulations with deep sound-speed perturbations (relative to a planeparallel quiet-Sun model) do not show a systematic frequency dependence at phase speeds above about $20 \mathrm{~km} \mathrm{~s}^{-1}$. However, shallow sound-speed perturbations, similar to those proposed to model the acoustic scattering properties of sunspots observed with Hankel analysis, produce systematic frequency dependence at these phase speeds. In both cases, positive travel-time shifts can be caused by positive sound-speed perturbations. The details of the travel-time shifts are, however, model dependent.
\end{abstract}

Keywords Helioseismology, forward modeling · Helioseismology, theory · Waves, propagation

\section{Introduction}

Local helioseismology is a promising tool for studying the subsurface nature of sunspots and active regions (for a recent review see Gizon and Birch, 2005). As discussed in Paper I

\author{
A.C. Birch $(\bowtie) \cdot$ D.C. Braun \\ NWRA, CoRA Division, Boulder, CO, USA \\ e-mail: aaronb@cora.nwra.com \\ S.M. Hanasoge \\ HEPL, Stanford University, Stanford, CA, USA \\ R. Cameron \\ Max-Planck-Institut für Sonnensystemforschung, 37191 Katlenburg-Lindau, Germany
}


of this series (Braun and Birch, 2008), surface-focusing Helioseismic Holography (hereafter HH; Lindsey and Braun, 1990; Braun and Lindsey, 2000) is one particular method for measuring the time required for waves to travel from one location on the solar surface to another. Surface-focusing $\mathrm{HH}$ is functionally very similar to surface-focusing time-distance helioseismology (Duvall et al., 1993). Both methods measure the correlation between the solar oscillations at a point on the solar surface and the oscillations seen in a surrounding annulus (or fraction of an annulus) also at the solar surface. The time required for waves to travel between the central point and the surrounding annulus is extracted from this correlation. These wave travel times are measurably different in active regions and sunspots than in the quiet Sun (e.g., Duvall et al., 1996; Chou, Sun, and Chang, 2000; Braun and Lindsey, 2000; Zhao and Kosovichev, 2003).

As was shown by Chou (2000), Braun and Lindsey (2000), Braun and Birch (2006), and in more detail in Paper I, travel-time shifts (relative to quiet-Sun averages) in active regions show a dependence on the temporal frequency of the waves used in the data analysis. Braun and Birch (2006) suggested that this frequency dependence may be the result of near-surface changes, relative to quiet Sun, in wave propagation properties.

In this paper, we study relatively simple, toy models to determine the frequency dependence of the travel-times shifts caused by local changes in background sound speed. Our approach is to carry out numerical simulations of the propagation of small-amplitude waves through heterogeneous background models. Wave travel times are then measured from these artificial wavefields by using surface-focusing $\mathrm{HH}$. Using this approach, we can study the frequency dependence of wave travel times for various types of local variations in sound speed.

We will show, using simulations carried out with two different codes, that shallow (depth scale of one $\mathrm{Mm}$ ) sound-speed perturbations (relative to a horizontally uniform background model) lead to frequency-dependent travel-time shifts. In contrast, a deep sound-speed perturbation (depth scale of ten $\mathrm{Mm}$ ) does not cause a strong systematic frequency dependence in travel-time shifts for phase speeds above about $20 \mathrm{~km} \mathrm{~s}^{-1}$. In addition, we show the nonintuitive result that increases in sound speed can, in the numerical simulations, in some cases induce increased apparent wave travel times.

\section{Numerical Simulations}

In this work, we employ the two separate numerical simulations codes: $i$ ) the code introduced by Hanasoge, Duvall, and Couvidat (2007) (hereafter referred to as H07) and ii) the SLiM code (Cameron, Gizon, and Daiffallah, 2007; Cameron, Gizon, and Duvall, 2008).

Both the H07 and SLiM codes compute the propagation of small-amplitude waves (both $p$ and $f$ modes) through arbitrary inhomogeneous background models (i.e., specifications for the nonwave components of pressure, density, temperature, etc.). In both codes, the waves are excited by near-surface stochastic sources that are statistically homogeneous in the horizontal directions. The H07 code employs absorbing side boundary conditions, whereas those of the SLiM are periodic.

In the H07 code, wave damping in the domain is due predominantly to the dissipative terms associated with the numerical scheme; we do not introduce phenomenological damping terms in the equations (unlike, e.g., Gizon and Birch, 2002, where wave dissipation is explicitly added to the equations of motion). The computational domain consists of $256 \times 256$ grid points horizontally and 300 grid points in depth and covers a region of $200 \times 200 \mathrm{Mm}^{2}$ horizontally and $35.8 \mathrm{Mm}$ in depth. The grid spacing is uniform in the horizontal directions 
and in the vertical direction is proportional to the local sound speed. Details of the numerical method are described by Hanasoge, Duvall, and Couvidat (2007) and code verification tests are discussed by Hanasoge (2007).

In the SLiM code, wave damping is explicitly modeled; the details are described by Cameron, Gizon, and Duvall (2008). The simulations described in this paper are two dimensional with 200 grid points in the horizontal direction and 558 grid points in the vertical direction. The simulations cover a region $146 \mathrm{Mm}$ wide and $12.5 \mathrm{Mm}$ deep. The grid spacing is uniform in both the horizontal and the vertical directions. Cameron, Gizon, and Duvall (2008) give details of the numerical methods.

We use both simulation codes to compute the propagation of waves through models that consist of a horizontally homogeneous background component intended to represent quiet Sun plus a localized enhancement of the sound speed intended as a proxy "sunspot." In the H07 code, for the "quiet Sun" component we use a simple model that consists of a truncated polytrope with an overlying isothermal atmosphere. The truncated polytrope is described by $g=2.775 \mathrm{~m} \mathrm{~s}^{-2}, m=2.64, z_{0}=345 \mathrm{~km}$, and $\rho_{0}=10^{-7} \mathrm{~g} \mathrm{~cm}^{-3}$ in the notation of Bogdan et al. (1996). The overlying isothermal atmosphere is chosen to extend $1.5 \mathrm{Mm}$ above the top of the truncated polytrope and is such that the sound speed, pressure, adiabatic exponent, and density are continuous across the boundary with polytrope. In the SLiM code, for the "quiet Sun" we use the stabilized model S described by Cameron, Gizon, and Duvall (2008).

In the region of enhanced sound speed we follow Fan, Braun, and Chou (1995) and introduce an axisymmetric change $(\delta c)$ in the sound speed of the form

$$
\delta c^{2}(r, z)=\epsilon c_{0}^{2}(z) \exp \left[-r^{2} / R^{2}\right] \exp \left[-z^{2} / D^{2}\right],
$$

where $r$ is distance from the symmetry axis of the sunspot, $z$ is depth with the model photosphere located at $z=0, c_{0}$ is the sound speed in the quiet-Sun model, $R$ is the horizontal length scale, $D$ is the depth scale, and $\epsilon$ is the fractional amplitude of the perturbation of the square of the sound speed. In the two-dimensional calculations, the variable $r$ can be understood as $|x|$, where $x$ is the horizontal coordinate.

These toy models are intended only to investigate the role of the depth scale $(D)$ in determining the frequency dependence of the resulting travel-time shifts. We emphasize that this simple model is not intended to capture the full physics of wave interactions with sunspots (e.g., Spruit and Bogdan, 1992; Cally, 2000; Cally, Crouch, and Braun, 2003; Crouch and Cally, 2003).

In this paper we describe six numerical experiments, three for each simulation code. The first is a case without a "sunspot" region (i.e., $\epsilon=0$ ). The second case is $D=10 \mathrm{Mm}$, $R=20 \mathrm{Mm}$, and $\epsilon=0.1$; this case will be referred to as the deep case. The final case has $D=1 \mathrm{Mm}, R=20 \mathrm{Mm}$, and $\epsilon=0.15$ for $\mathrm{H} 07$ and $\epsilon=0.3$ for SLiM and will be referred to as the shallow case. The different choices for $\epsilon$ for the $D=1 \mathrm{Mm}$ cases were made to ensure that the travel-time shifts have similar magnitudes.

For each case, the output of the numerical wave-propagation simulation is, at each time step, a map of the vertical velocity at a height of $200 \mathrm{~km}$ above the photosphere. These maps are collected into data cubes with a cadence of one minute. The data cubes are then subjected to data analysis methods to determine travel-time shifts.

\section{Observations}

In this section we describe the measurement of travel times from the simulated data cubes (Section 2). As in Paper I, we apply surface-focusing HH. Also as in Paper I, we use both 

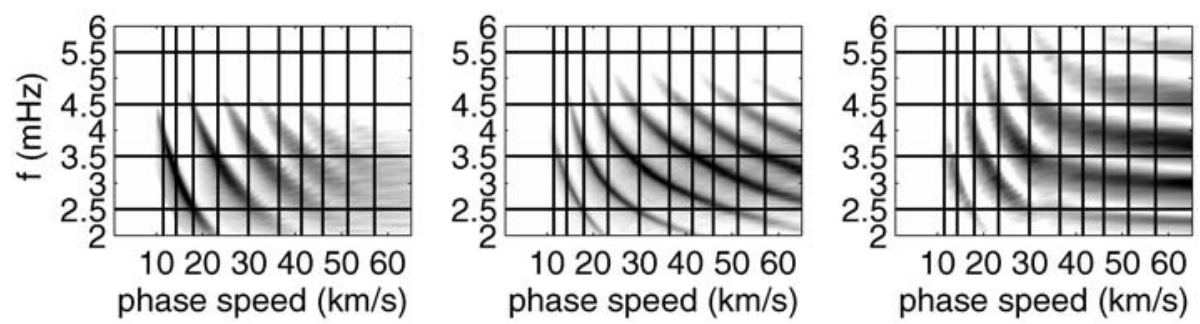

Figure 1 Power spectra of the simulated data from H07 (left), from MDI full-disk data (middle), and from SLiM (right) as functions of phase speed and temporal cyclic frequency. The horizontal black lines show the frequency bandpass filters. The vertical black lines show where the square of each of the phase-speed filters reaches a value of one half.

phase-speed filters (Duvall et al., 1997) and frequency bandpass filters (see, e.g., Paper I) to isolate particular regions in horizontal-wavenumber and frequency space. This filtering is useful as it allows us to measure the variation of travel times with frequency and phase speed. In this paper we use the same filter parameters as in Paper I.

One complication in the analysis of the simulated data is that the power spectra of the simulated data are not the same as the power spectrum seen in SOHO/MDI data (Scherrer et al., 1995). Figure 1 shows a comparison between the power spectra of the simulated data for the cases without a sound-speed perturbation and an example power spectrum from the MDI full-disk data.

For the H07 code, the ridges in the simulated data are not in the same places as those in the MDI data. Two possible causes for this are that $i$ ) the upper boundary condition of the simulation (the zero-velocity upper boundary condition imposed by the upper sponge layer) is not solar-like and ii) the simple truncated polytrope with an overlying isothermal atmosphere is not sufficiently solar-like. As the resonant frequencies of the simulated data are not the same as those seen in the MDI data, the data analysis tools of Paper I cannot be applied without modification, as will be described later in this section.

For the SLiM code, the ridge positions, at phase speeds below about $30 \mathrm{~km} \mathrm{~s}^{-1}$, are in reasonable agreement with those of the MDI data. Above this phase speed the ridge positions begin to deviate from those seen in the data; in particular the ridges become flat. This is likely due to the influence of the bottom boundary, where the sound speed is about $42 \mathrm{~km} \mathrm{~s}^{-1}$.

Here, for the analysis of the both the H07 and SLiM simulations, we modify the measurement procedure described in Paper I by recomputing the holography Green's functions and also recomputing the pupil sizes. The holography Green's functions are computed by using the ray approximation method of Lindsey and Braun (2000), using the known background stratification from the numerical simulations (Section 2). Pupil sizes are computed as described in Paper I: The inner and outer radii of the pupils are determined from ray tracing based on the parameters of the phase-speed filters.

Werne, Birch, and Julien (2004) introduced the technique of noise subtraction to improve the signal-to-noise ratio of the travel times measured from the simulations. In the simulations, the dominant noise source is realization noise (i.e., noise from the stochastic nature of the wave excitation). This noise can be greatly reduced by carrying out simulations with and without the perturbations to the background model (in the case discussed here the local change in the sound speed) but with the same realization of the wave forcing function. By subtracting the wave travel times seen in the simulation with an unperturbed background model from those measured from the simulation with the perturbed background model, travel-time shifts that essentially do no contain realization noise can be measured. Here, 
Figure 2 Travel-time shifts for the shallow sound-speed perturbations (left column) and the deep perturbation (right column) for three different frequency bandpass filters for the H07 simulations. The gray scale for the shallow perturbation has been increased by a factor of seven for ease of comparison. The same phase-speed filter (filter D; see Paper I) was used in all cases. The top row shows maps for a 1-mHz bandpass filter centered at $3 \mathrm{mHz}$. The middle and bottom rows show the cases of frequency filters centered at 4 and $5 \mathrm{mHz}$, respectively.

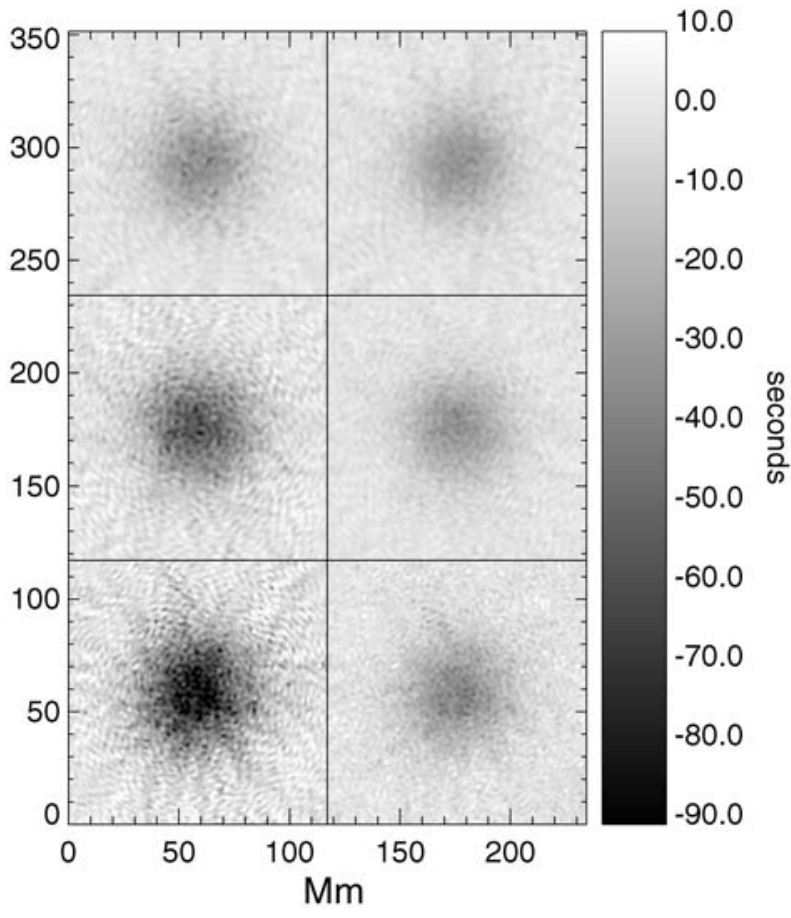

we carry out noise subtraction by computing the symmetric phases using surface-focusing $\mathrm{HH}$ (see Paper I for details) for each simulated data cube. We then subtract the phases seen in the "quiet Sun" simulation from those measured in the shallow $(D=1 \mathrm{Mm})$ and deep ( $D=10 \mathrm{Mm}$ ) cases to measure phase shifts for these two cases. These phase shifts are converted to travel-time shifts, relative to the "quiet Sun," as in Paper I.

\section{Results}

Using the filters parameters described in Paper I, the holography Green's functions and pupils described in Section 3, and the noise subtraction method, we computed travel-time shifts from the numerical data cubes described in Section 2. All of the results shown in this paper are for shifts in the mean travel times (i.e., the average of the ingoing and outgoing travel times). Figure 2 shows some example maps of travel-time shifts from the H07 simulations, all computed using the same phase-speed filter (Filter D; see Paper I). For this particular phase-speed filter, in the deep case $(D=10 \mathrm{Mm})$ the maps of travel times depend only weakly on frequency. For the shallow $(D=1 \mathrm{Mm})$ case, the magnitude of the travel-time shifts increases with increasing frequency.

\subsection{Frequency Variations at Fixed Phase Speed}

Figure 3 shows travel-time shifts, averaged over a disk of $39 \mathrm{Mm}$ diameter centered on the sound-speed perturbation, for the shallow and deep cases for the H07 simulations. For the case of a deep perturbation, at fixed phase speed the travel-time shifts show little systematic variation with frequency at phase speeds above about $20 \mathrm{~km} \mathrm{~s}^{-1}$. However, at most phase 
speeds, the travel-time shifts for the shallow case show an essentially monotonic increase in amplitude with frequency at fixed phase speed. This frequency dependence suggests that the height of the upper turning point of the waves is important. Figure 3 supports the suggestion of Braun and Birch (2006) that strong frequency variations are not consistent with deep sound-speed variations.

The variations of the travel-time shifts with phase speed (Figure 3) are dominated by systematic effects, not random noise. The noise level, estimated by dividing the spatial averaging region in half, is smaller than twice the size of the symbols in Figure 3. These systematic variations of the travel-time shifts with phase speed are likely due to the details of the way in which the ridges in the power spectrum are weighted by the phase-speed filters (Figure 1).

Figure 3 also shows that for some combinations of frequencies and phase-speeds we measure positive travel-time shifts. In the ray approximation (e.g., Equation (14) of Kosovichev and Duvall, 1997) as applied to the background stratification employed in the simulations discussed in this paper (in which the square of the acoustic-cutoff frequency is never negative), increases in sound speed cannot cause positive travel-time shifts. The travel times shown in Figure 3 are not consistent with the ray approximation.

Figure 4 shows travel-time shifts as functions of phase speed and temporal frequency from the two-dimensional SLiM simulations. As in the H07 case, for phase speeds above $20 \mathrm{~km} \mathrm{~s}^{-1}$ there is no clear frequency dependence in the deep $(D=10 \mathrm{Mm})$ case. For most phase speeds in the shallow $(D=1 \mathrm{Mm})$ case, the travel-time shifts at $3 \mathrm{mHz}$ are smaller than the travel-time shifts at the other frequencies. Unlike in the $\mathrm{H} 07$ case, the 4- and 5-mHz travel-time shifts are quite similar. As with the $\mathrm{H} 07$ results, at the lowest phase speeds the travel-time shifts are positive. Again, this is notable as the simulations contain only an increase in the sound speed.

\subsection{Sensitivity to Choice of Filter}

The presence of positive travel-time shifts at the lowest phase speeds is reminiscent of the measurements made with MDI data shown in Paper I. Paper I demonstrated that, for the lowphase speed measurements, the measured travel-time shifts depend critically on the detailed form of the data analysis filters. The same is true for the travel-time shifts measured from the simulated data shown here.

Figure 5a shows the power spectrum corresponding to the simulated vertical velocity (for the $D=10 \mathrm{Mm} \mathrm{H07}$ case) after it has been filtered using filter 4B (which is the second phase speed filter with a frequency bandpass filter centered at $4 \mathrm{mHz}$; see Paper I for a complete description of filters and naming conventions). For this case, the filtered power consists of the $p_{1}$ ridge, as well as power beneath the ridge, but above the $f$-mode ridge. The origin of this sub- $p_{1}$ power in the simulation is not known. Figure $5 \mathrm{~b}$ shows the change in the power spectrum caused by the deep sound-speed perturbation. As expected, the increase in the sound speed causes the $p_{1}$ ridge to move upward. In addition, the sub- $p_{1}$ power has changed. Figure $5 \mathrm{c}$ shows the map of travel-time shifts for this case.

Figure $5 \mathrm{~d}$ shows the power spectrum of the simulated vertical velocity (again for the deep H07 case) after filter 4B, as well as an additional filter $F$ to remove the sub- $p_{1}$ power, has been applied. The filter $F$ we choose to be a function of horizontal wavenumber $k$ and frequency $v$ such that, for each $k, F(k, v)=1$ when $v$ is greater than $0.05 \mathrm{mHz}$ less than the $p_{1}$ resonance frequency at $k, F(k, v)=0$ when $v$ is less than $0.25 \mathrm{mHz}$ less than the $p_{1}$ resonance frequency at $k$, and $F$ varies as a raised cosine of $v$ in between these two frequencies. Figure 5e shows the change in the filtered power spectrum caused by the 

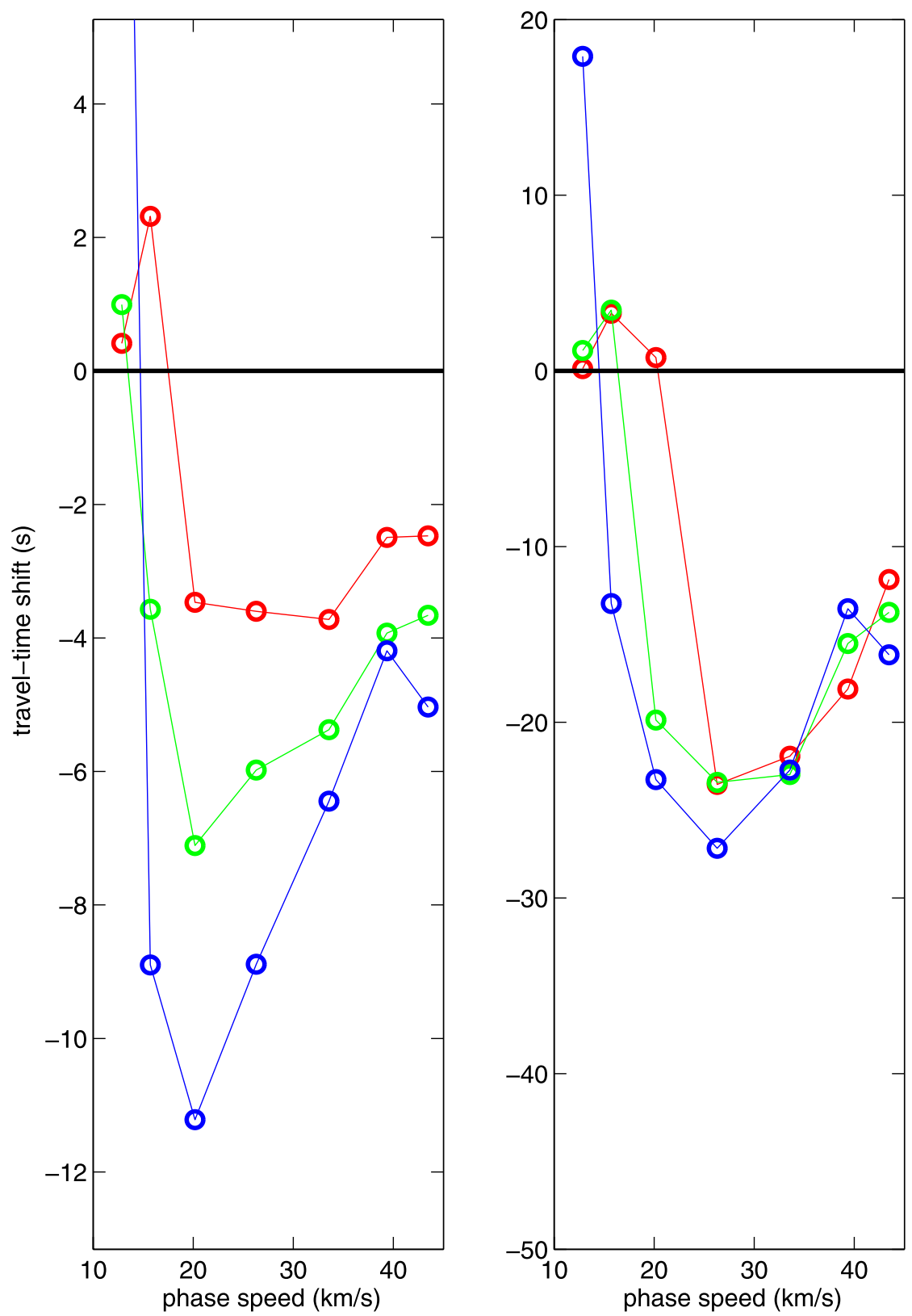

Figure 3 Travel-time shifts from the H07 code, averaged over a disk of $39 \mathrm{Mm}$ diameter centered on the sound-speed perturbation, for the shallow case $(D=1 \mathrm{Mm}$, left $)$ and the deep case $(D=10 \mathrm{Mm}$, right $)$ for three frequency bandpass filters $[3 \mathrm{mHz}$ (red), $4 \mathrm{mHz}$ (green), and $5 \mathrm{mHz}$ (blue)] as functions of horizontal phase speed. In the deep case, there is no clear dependence of the travel-time shifts on frequency at phase speeds above $20 \mathrm{~km} \mathrm{~s}^{-1}$. In the shallow case, at most phase speeds, the travel-time shifts increase in magnitude with increasing frequency. The error estimates are all smaller than twice the size of the symbols on the plots. In the left-hand plot, there is one point that is located off the top of the plot with a value of $16.6 \mathrm{~s}$ at a phase speed of $12.9 \mathrm{~km} \mathrm{~s}^{-1}$ for the frequency bandpass centered on $5 \mathrm{mHz}$. 

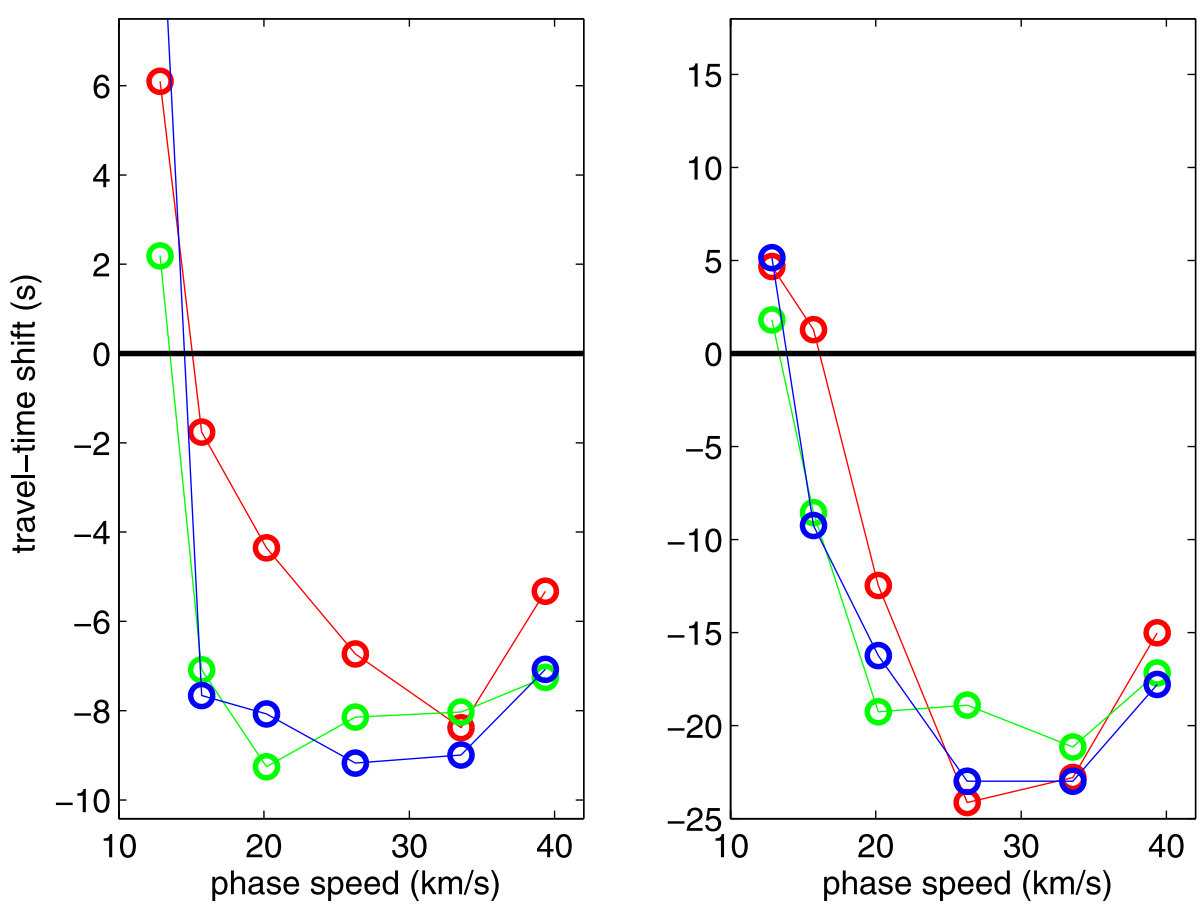

Figure 4 Travel-time shifts from the SLiM code (averaged over a rectangle of dimensions of $72 \times 200 \mathrm{Mm}$ with the short side centered on the sound-speed perturbation and the long side in the direction in which the sound-speed perturbation is constant) for the shallow case $(D=1 \mathrm{Mm}$, left $)$ and the deep case $(D=10 \mathrm{Mm}$, right) for three frequency bandpass filters $[3 \mathrm{mHz}$ (red), $4 \mathrm{mHz}$ (green), and $5 \mathrm{mHz}$ (blue)] as functions of horizontal phase speed. In the deep case, there is no clear dependence of the travel-time shifts on frequency at phase speeds above $20 \mathrm{~km} \mathrm{~s}^{-1}$. In the shallow case, the travel-time shifts at $3 \mathrm{mHz}$ tend to be smaller than those at $4 \mathrm{mHz}$ and $5 \mathrm{mHz}$. The maximum error estimates are smaller than twice the size of the symbols on the plots. In the left-hand plot, there is one point that is located off the top of the plot with a value of 17.9 seconds at a phase speed of $12.9 \mathrm{~km} \mathrm{~s}^{-1}$ for the frequency bandpass centered on $5 \mathrm{mHz}$.

introduction of the deep sound-speed perturbation. In this case, the change in the power spectrum is dominated by the change in the ridge position. The change in the sub- $p_{1}$ power has been removed by the filter $F$. Figure $5 \mathrm{f}$ shows the travel-time map corresponding to filter 4B used in conjunction with the filter $F$ described in this paragraph. Notice that the sign of the travel-time shifts is opposite to that seen in Figure $5 \mathrm{c}$, which was made using only filter $4 \mathrm{~B}$.

A comparison of Figures $5 \mathrm{c}$ and $5 \mathrm{f}$ shows that, at least for the case of measurements made with filter 4B, the travel-time shifts measured from the simulated data are sensitive to the presence of the sub- $p_{1}$ power in the wavefield. This situation is qualitatively similar to what is observed in the MDI data (see Paper I), in which the choice to use phase-speed filters or ridges filters can change the sign of the measured travel-time shifts.

\section{Conclusions}

We have used two different simulation codes to study the travel-time shifts caused by local changes in sound speed. Both the H07 and SLiM shallow $(D=1 \mathrm{Mm})$ cases show a clear 
Figure 5 A comparison of a measurement for the $D=10 \mathrm{Mm}$ H07 case with filter 4B (left column) and filter $4 \mathrm{~B}$ plus a filter to remove the sub- $p_{1}$ power (right column). (a) The power spectrum of the simulated data after applying filter 4B. (b) The change in the power spectrum caused by the deep sound-speed perturbation. (c) The travel-time map corresponding to filter $4 \mathrm{~B}$.

(d) The simulated power spectrum after applying both filter $4 \mathrm{~B}$ and the sub- $p_{1}$ filter $F$ (see text). (e) The change in the filtered power, with both filters $4 \mathrm{~B}$ and $F$. (f) The travel-time map resulting from employing both filters $4 \mathrm{~B}$ and $F$. The gray scale is such that white indicates an increase in power in panels $b$ and e. Notice that the travel-time maps in panels $\mathrm{c}$ and $\mathrm{f}$ show travel time shifts of dominantly opposite sign.
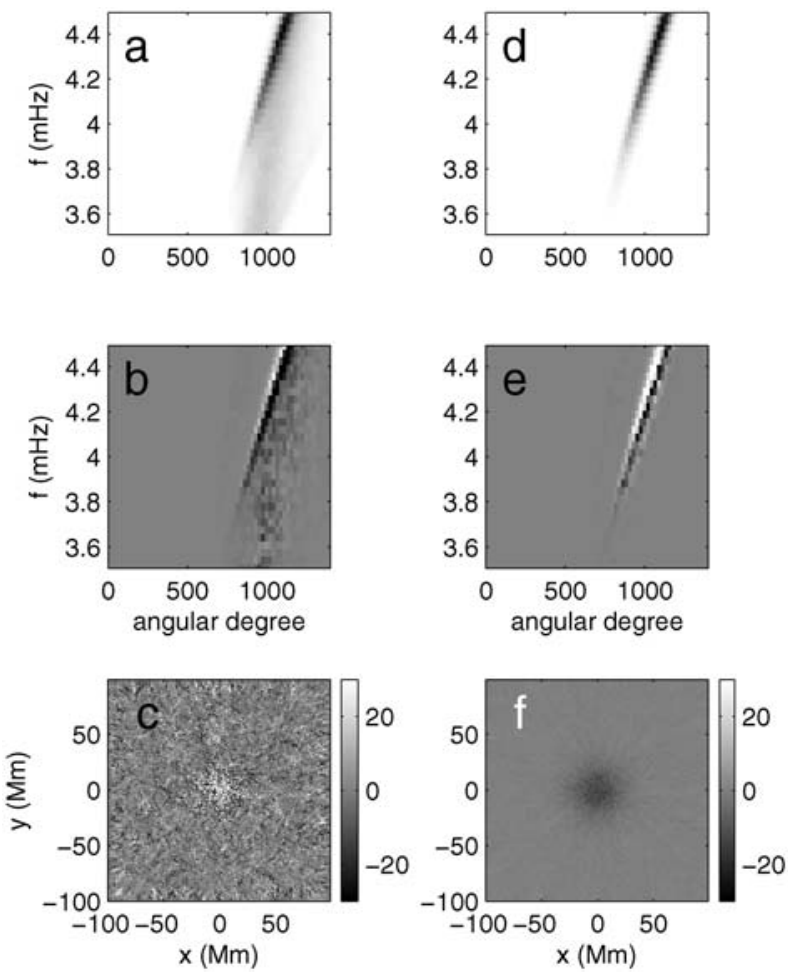

pattern that the travel-time shifts, for phase speeds greater than about $20 \mathrm{~km} \mathrm{~s}^{-1}$, at $3 \mathrm{mHz}$ are smaller in amplitude that those at $5 \mathrm{mHz}$. This pattern is similar to the pattern seen in the Sun (see Paper I). This pattern is not seen in the travel-time shifts caused by deep soundspeed perturbations. These qualitative results support the suggestion of Braun and Birch (2006) that the strong frequency dependence in observed travel-time shifts in sunspots may be consistent with a near-surface change in wave propagation properties.

We have also shown that, in the numerical simulations presented here, local increases in sound speed may introduce positive travel-time shifts. The result is in direct contradiction with the ray approximation. We have shown that this result, however, depends critically on the choice of data analysis filter. The dependence of travel-time shifts on the data analysis filters has been explored in more detail by Braun and Birch (2008) and Thompson and Zharkov (2008). Note that we are not suggesting that any particular type of filter produces nonsensible results. The dependence of travel-time shifts on the choice of filter function does, however, highlight the need for future modeling efforts.

The differences in the results obtained using the two different simulation codes (those of Hanasoge, Duvall, and Couvidat, 2007, and Cameron, Gizon, and Duvall, 2008, which use two different methods of producing convectively stable background solar models) show that the choice of background solar model is important in determining travel-time shifts.

The simple toy models presented here are useful as they allow the study, in a qualitative sense, of one possible source of frequency dependence in wave travel times. The computation of wave propagation through realistic, magnetic sunspot models is needed before quantitative comparisons with observations can be made. 
Acknowledgements The work of A.C.B. and D.C.B. was supported by NASA Contract Nos. NNH04CC05C, NNH06CD84C, NNH05CC76C, and NNG07E151C and NSF Grant No. AST-0406225, along with a subcontract through the HMI Project at Stanford University awarded to NWRA. S.M.H. acknowledges support from HMI NAS5-02139. The artificial data from the H07 code were generated using the supercomputer Columbia.

Open Access This article is distributed under the terms of the Creative Commons Attribution Noncommercial License which permits any noncommercial use, distribution, and reproduction in any medium, provided the original author(s) and source are credited.

\section{References}

Bogdan, T.J., Hindman, B.W., Cally, P.S., Charbonneau, P.: 1996, Absorption of p-Modes by Slender Magnetic Flux Tubes and p-Mode Lifetimes. Astrophys. J. 465, 406.

Braun, D.C., Birch, A.C.: 2006, Surface effects in sunspot seismology. Astrophys. J. 647, 187.

Braun, D.C., Birch, A.C.: 2008, Surface-focused seismic holography of sunspots: I. Observations. Solar Phys. 251, 267. doi:10.1007/s11207-008-9152-5.

Braun, D., Lindsey, C.: 2000, Phase-sensitive holography of solar activity. Solar Phys. 192, 307.

Cally, P.S.: 2000, Modelling p-mode interaction with a spreading sunspot field. Solar Phys. 192, 395.

Cally, P.S., Crouch, A.D., Braun, D.C.: 2003, Probing sunspot magnetic fields with p-mode absorption and phase-shift data. Mon. Not. Roy. Astron. Soc. 346, 381.

Cameron, R., Gizon, L., Daiffallah, K.: 2007, SLiM: a code for the simulation of wave propagation through an inhomogeneous, magnetised solar atmosphere. Astron. Nachr. 328, 313.

Cameron, R., Gizon, L., Duvall, T.L. Jr.: 2008, Helioseismology of sunspots: confronting observations with three-dimensional MHD simulations of wave propagation. Solar Phys. 251, 291. doi:10.1007/s11207-008-9148-1.

Chou, D.Y.: 2000, Acoustic imaging of solar active regions - (Invited Review). Solar Phys. 192, 241.

Chou, D.Y., Sun, M.T., Chang, H.K.: 2000, A study of sunspots with phase time and travel time of p-mode waves in acoustic imaging. Astrophys. J. 532, 622.

Crouch, A.D., Cally, P.S.: 2003, Mode conversion of solar p modes in non-vertical magnetic fields - i. Twodimensional model. Solar Phys. 214, 201.

Duvall, T.L., Jefferies, S.M., Harvey, J.W., Pomerantz, M.A.: 1993, Time-distance helioseismology. Nature 362, 430 .

Duvall, T.L., D’Silva, S., Jefferies, S.M., Harvey, J.W., Schou, J.: 1996, Downflows under sunspots detected by helioseismic tomography. Nature 379, 235.

Duvall, T.L., Kosovichev, A.G., Scherrer, P.H., Bogart, R.S., Bush, R.I., de Forest, C., Hoeksema, J.T., Schou, J., Saba, J.L.R., Tarbell, T.D., Title, A.M., Wolfson, C.J., Milford, P.N.: 1997, Time-distance helioseismology with the MDI instrument: initial results. Solar Phys. 170, 63.

Fan, Y., Braun, D.C., Chou, D.Y.: 1995, Scattering of p-modes by sunspots. II. Calculations of phase shifts from a phenomenological model. Astrophys. J. 451, 877.

Gizon, L., Birch, A.C.: 2002, Time-distance helioseismology: the forward problem for random distributed sources. Astrophys. J. 571, 966.

Gizon, L., Birch, A.C.: 2005, Local helioseismology. Living Rev. Solar Phys. 2, 6. http://solarphysics. livingreviews.org/Articles//rsp-2005-6/.

Hanasoge, S.: 2007, Theoretical studies of wave propagation in the sun. PhD thesis, Stanford University.

Hanasoge, S.M., Duvall, T.L. Jr., Couvidat, S.: 2007, Validation of helioseismology through forward modeling: realization noise subtraction and Kernels. Astrophys. J. 664, 1234.

Kosovichev, A.G., Duvall, T.L. Jr.: 1997, Acoustic tomography of solar convective flows and structures. In: Pijpers, F.P., Christensen-Dalsgaard, J., Rosenthal, C.S. (eds.) SCORe'96: Solar Convection and Oscillations and their Relationship, Astrophysics and Space Science Library 225, 241.

Lindsey, C., Braun, D.: 1990, Helioseismic imaging of sunspots at their antipodes. Solar Phys. 126, 101.

Lindsey, C., Braun, D.C.: 2000, Basic principle of solar acoustic holography. Solar Phys. 192, 261.

Scherrer, P.H., Bogart, R.S., Bush, R.I., Hoeksema, J.T., Kosovichev, A.G., Schou, J., Rosenberg, W., Springer, L., Tarbell, T.D., Title, A., Wolfson, C.J., Zayer, I., MDI Engineering Team: 1995, The solar oscillations investigation - Michelson Doppler imager. Solar Phys. 162, 129.

Spruit, H.C., Bogdan, T.J.: 1992, The conversion of p-modes to slow modes and the absorption of acoustic waves by sunspots. Astrophys. J. 391, L109. 
Thompson, M.J., Zharkov, S.: 2008, Recent developments in local helioseismology. Solar Phys., $251,225$. doi:10.1007/s11207-008-9143-6.

Werne, J., Birch, A., Julien, K.: 2004, The need for control experiments in local helioseismology. In: Dansey, D. (ed.) Proceedings of the SOHO 14/GONG 2004 Workshop (ESA SP-559) Helio- and Asteroseismology: Towards a Golden Future SP-559, ESA, Noordwijk, 172.

Zhao, J., Kosovichev, A.: 2003, Helioseismic observation of the structure and dynamics of a rotating sunspot beneath the solar surface. Astrophys. J. 591, 446. 